\title{
DEFINING NORMAL SUBGROUPS OF UNIPOTENT ALGEBRAIC GROUPS
}

\author{
A. FAUNTLEROY ${ }^{1}$
}

ABSTRACT. Let $G$ be a connected unipotent algebraic group defined over the perfect field $k$. We show that polynomial generators $x_{1}, \cdots, x_{n}$ for the ring $k[G]$ can be chosen so that if $N$ is any connected normal $k$ closed subgroup of $G$, then $I(N)$ can be generated by codim $N p$-polynomials in $x_{1}, \cdots, x_{n}$ where $p=$ char $k$. Moreover $k[G / N]$ can also be generated as a polynomial algebra over $k$ by p-polynomials.

Introduction. These results are essentially an extension of a theorem of Rosenlicht [4, Theorem 1].

We use the notation and conventions of [1] throughout this paper.

Recall that a $p$-polynomial in $k[T]$ is a linear form if $p=0$ and a polynomial all of whose exponents are powers of $p$ if $p>0$. A $p$-polynomial in $k\left[x_{1}, \cdots, x_{n}\right]$ is a sum of $p$-polynomials in each of the single variables $x_{1}$, $\cdots, x_{n}$. A function $f \in k[G]$ will be called additive if $f(a b)=f(a)+f(b)$ for all closed points $a, b$ in $G$.

1. Frattini coordinates. Let $G$ be a unipotent algebraic group. The Frattini subgroup of $G$ is the intersection of all closed subgroups of codimension one. We shall denote this group by $\operatorname{Fr}(G)$.

Proposition 1. If $G$ is a unipotent algebraic group then $F r(G)$ is a closed characteristic subgroup of $G$. If $G$ is connected and defined over the perfect field $k$, then $\operatorname{Fr}(G)$ is connected and defined over $k$. Moreover in the connected case $G / F r(G)$ has the structure of a vector group (over $k$ if $G$ is defined over $k$ ) and is the maximal such quotient.

Proof. The first assertion is immediate. Let $H \subset G$ be a closed subgroup of $G$ of codimension one. Since $G / H \simeq G_{a}, H$ contains the commutator subgroup of $G$ and the group generated by the $p$ th powers of the elements of $G$. It follows that $\operatorname{Fr}(G)$ also contains these subgroups.

Thus $G / F r(G)$ is connected, commutative and of exponent $p$ hence by

Received by the editors December 28, 1973.

AMS(MOS) subject classifications (1970). Primary 14L10, $20 \mathrm{G} 15$.

Key words and phrases. Unipotent group, p-polynomial, Frattini coordinates.

1 This work was partially supported by NSF grant GP 38916. 
[3, Proposition 2] has the structure of a vector group. If $N \subset G$ is any normal subgroup such that $G / N$ is isomorphic to $G_{a}^{r}$ for some integer $r$, then consider the natural map $G \rightarrow G / N \simeq G_{a}^{r}$ followed by projection $\Pi_{i}$ onto each factor, $i=1,2, \cdots, r$. Each $\Pi_{i}$ is a homomorphism with kernel say $H_{i}$ and $\bigcap H_{i}=N$. Since $H_{i}$ has codimension one, $N \supset \operatorname{Fr}(G)$ and $G / N$ is an image of $G / F r(G)$ which establishes the maximality assertion.

As for rationality and connectedness, let $N$ be the closed normal subgroup generated by the commutator subgroup and $p$ th powers of the elements of $G$. Then $N \subset \operatorname{Fr}(G), N$ is connected and $G / N$ has the structure of a vector group [3, Proposition 2] so $N=\operatorname{Fr}(G)$. Since $N$ is defined over $k$ so is $G / N[1,6.8]$. This completes the proof.

Now let $G$ be a connected unipotent algebraic group and $\operatorname{Fr}(G)$ the Frattini subgroup of $G$. If $G$ is defined over the perfect field $k$ then by [4, Corollary 2 of Theorem 1], $k[G]$ is $k$-isomorphic to $k[G / F r(G)] \otimes k[F r(G)]$. Let $x_{1}, \cdots:, x_{r}$ be additive coordinates for the vector $k$-group $G / F r(G)$ (cf. [3, $\S 1])$. Then $k[G]=k\left[x_{1}, \cdots, x_{r}\right] \otimes k[F r(G)]$. By the proposition $F_{1}=F r(G)$ is again connected and defined over $k$ and we may continue this process until we arrive at a complete set of polynomial generators for $k[G]$. A set of polynomial generators $x_{1}, \cdots, x_{n}$ obtained in this way will be called a set of Frattini coordinates for $G$.

In case $G$ itself has the structure of a vector group, these coordinates have essentially been studied by Rosenlicht [3], [4] and Tits [7, III, 3.3]. In particular the following proposition is easily deduced from their results.

Proposition 2. Let $V$ be a connected unipotent algebraic group defined over the perfect field $k$. Suppose $V$ has the structure of a vector group over $k$ and $x_{1}, \cdots, x_{n}$ are Frattini coordinates for $V$. Then

(i) if $W$ is any $k$-closed subgroup of $V$ then $I(W)$ is generated by codim $W$ p-polynomials in $x_{1}, \cdots, x_{n}$;

(ii) the Frattini coordinates of $k[V / W] \subset k[V]$ are p-polynomials in the Frattini coordinates of $V$.

Now let $G$ be any connected unipotent group defined over the perfect field $k$. Let $N \subset \operatorname{Fr}(G)=F$ be a $k$-closed normal subgroup of $G$. Then since $G / N \simeq G / F \times F / N$ we have $k[G / N] \simeq k[G / F] \otimes k[F / N]$. It follows from (ii) above that if $F r(F)=e$ then a set of Frattini coordinates for $G / N$ may be taken to be $p$-polynomials in any fixed set of Frattini coordinates of $G$.

Theorem. Let the connected unipotent algebraic group $G$ be defined

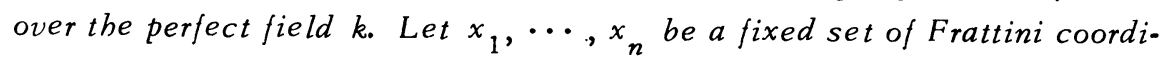


nates for $G$. Suppose $Z$ is a closed connected central one dimensional sub. group of $G$ defined over $k$. Then there exists a set of Frattini coordinates of $G / Z$ in $R=k[G / Z] \subset k[G]$ which consists of p-polynomials in $x_{1}, \cdots, x_{n}$.

Proof. Let $F_{0}=G \supset F_{1}=F r(G) \supset \cdots \supset F_{S} \supset e$ be the Frattini series of $G$. We argue by induction on the length, $s$, of the series. Thus, suppose $s=1$. If $Z \subset F_{1}$ then $G / Z \simeq G / F \times F / Z$, hence $k[G / Z] \simeq k[G / F] \otimes k[F / Z]$. But by the remarks above, $k[G / Z]$ has $p$-polynomials in $x_{1}, \cdots, x_{n}$ as Frattini coordinates.

If $Z \cap F_{1}$ is finite we distinguish two cases.

Case 1. $Z \cap F_{1}=e$. Then $Z F_{1} / F_{1}$ is a direct factor of $G / F_{1}$ and is not equal to $G / F_{1}$ since $Z$ is contained in a subgroup of codimension one.

Let $L \supset F_{1}$ be a connected $k$-closed subgroup of $G$ such that $L / F_{1}$ is a complement of $Z F_{1} / F_{1}$ in $G / F_{1}[3$, Proposition 1]. Then $\operatorname{codim} L=1$, hence $L$ is normal in $G$. If $N=L \cap Z$ then $N F_{1} / F_{1}=e$, hence $N \subset Z \cap F_{1}$. Thus $L \cap Z=e$ and clearly $L Z=G$.

Consider the commutative diagram

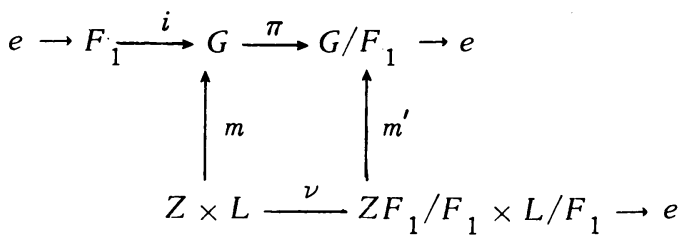

where $i$ is inclusion, $\pi$ the quotient morphism, $m$ and $m^{\prime}$ multiplication, and $\nu=\left.\pi\right|_{Z} \times\left.\pi\right|_{L}$.

Te obtain a commutative diagram of Lie algebras:

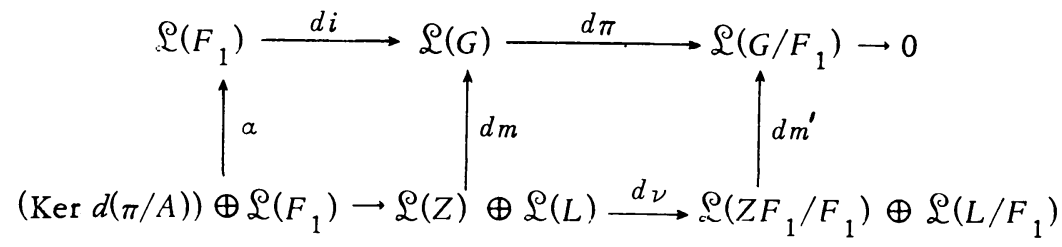

Since $\alpha$ and $d m^{\prime}$ are surjective so is $d m$. Thus $d m$ is an isomorphism and $m$ is separable. It follows from [1, Chapter II, 6.1] that $m: Z \times L \rightarrow G$ is an isomorphism.

Now choose new Frattini coordinates $y_{1}, \cdots, y_{r}, x_{r+1}, \cdots, x_{n}$ such that $V\left(y_{1}, \cdots, y_{r}\right)=F_{1}$ and $V\left(y_{1}\right)=L$. Then $y_{1}, \cdots, y_{r}$ are $p$-polynomials in $x_{1}, \cdots, x_{r}$ and $k[G / Z]=k[L]=k\left[y_{2}, \cdots, y_{r}, x_{r+1}, \cdots, x_{n}\right]$.

Case 2. $\Lambda=Z \cap F \neq e$. Then in $G / \Lambda$ we have the conditions of Case 1 . 
Hence $k[G / Z]=k[G / \Lambda / Z / \Lambda] \subset k[G / \Lambda]$ is generated by $p$-polynomials in any set of Frattini coordinates of $G / \Lambda$. But we may assume these last are $p$-polynomials in $x_{1}, \cdots, x_{n}$. Hence $k[G / Z]$ has a set of Frattini coordinates consisting of $p$-polynomials in $x_{1}, \cdots, x_{n}$ and the case $s=1$ is done.

If $s>1$ we form the chain

$$
G \supset F_{1}^{\prime} \supset F_{1} \supset F_{2}^{\prime} \supset F_{2} \supset \cdots \supset F_{l}^{\prime} \supset Z \supset e
$$

where $F_{i}^{\prime} / Z$ is the $i$ th term in the Frattini series of $G / Z$, and $F_{l}^{\prime} / Z$ has the structure of a vector group over $k$. Each $F_{i}^{\prime}$ may be taken to be connected, closed and defined over $k$.

Suppose $l \geq 2$. Then $F_{1} \supset Z$. By induction $k\left[F_{1} / Z\right] \subset k\left[F_{1}\right]$ has a set of Frattini coordinates which consists of $p$-polynomials in $x_{r+1}, \cdots, x_{n}$. It then follows as before from the isomorphism $k[G / Z] \simeq k\left[G / F_{1}\right] \otimes k\left[F_{1} / Z\right]$ that $G / Z$ has the desired property.

If $l=1$ then $F_{1}^{\prime} / Z$ has the structure of a vector group. But then $F_{1} \subset$ $F_{1}^{\prime}$ and if $Z \subset F_{1}$ we are done arguing as above. If not, $Z \cap F_{1}$ is finite and $F_{1} \rightarrow F_{1} / Z \cap F_{1}$ is an isogeny whose image is a vector group. Hence $F_{1}$ itself has the structure of a vector group so $s=1$ a contradiction. This completes the proof.

Corollary 1. Let $G$ be a connected unipotent group defined over the perfect field $k$. Let $N$ be a connected closed normal subgroup of $G$ also de. fined over $k$. Then the Frattini coordinates of $G / N$ in $k[G / N]$ can be taken to be p polynomials in any fixed set of Frattini coordinates of $G$.

Proof. Any connected closed subgroup normal in $G$ and defined over $k$ contains a central connected subgroup of dimension one defined over $k$ by [5]. The corollary now follows by induction on the dimension of $N$.

Corollary 2. Suppose $G$ and $k$ are as above. Then every normal closed connected subgroup $N$ of $G$ which is defined over $k$ can be defined by $d=$ $\operatorname{codim} N$ p-polynomials in $x_{1}, \cdots, x_{n}$. Moreover these may be chosen so as to generate the ideal $I(N)$.

Proof. We have $G / N \times N \simeq G$ and by Corollary $1, k[G / N]$ is generated by $p$-polynomials in a fixed set of Frattini coordinates for $G$. Say $k[G / N]=$ $k\left[f_{1}, \cdots, f_{d}\right] \subset k[G]$ where $d=\operatorname{codim} N$ and the $f_{i}, i=1, \cdots, d$, are $p$-polynomials in $x_{1}, \cdots, x_{n}$. Then each $f_{i}$ is constant on the fibres of $\pi: G \rightarrow G / N$ and vanishes on $N$.

Since $k[G / N] \rightarrow k[G / N] \otimes k[N]=k[G]$ is a polynomial extension by $[4$, 
Corollary 1 of Theorem 1$]$ the ideal $\left(f_{1}, \cdots, f_{d}\right) k[G]$ is prime in $k[G]$. Hence $I(N)=\left(f_{1}, \cdots, f_{d}\right) k[G]$.

Remarks. 1. Corollary 2 is false without the assumption of normality on $N \subset G$. Consider the following example suggested by Rosenlicht.

Let $G$ be the group of $3 \times 3$ upper triangular unipotent matrices

$$
G=\left\{\left[\begin{array}{lll}
1 & x & z \\
0 & 1 & y \\
0 & 0 & 1
\end{array}\right]: x, y, z \in K, \operatorname{char} K \neq 2\right\} .
$$

Let $x, y$ and $z$ be the obvious Frattini coordinates. Then

$$
N=\left\{\left[\begin{array}{ccc}
1 & t & t^{2} / 2 \\
0 & 1 & t \\
0 & 0 & 1
\end{array}\right]: t \in K\right\}
$$

is a connected subgroup of $G$. The ideal $I(N)=\left(x-y, z-x^{2} / 2\right)$ is clearly not generated by two $p$-polynomials.

Moreover if $H \subset G$ is the subgroup defined by $x-y=0$ and $N \subset H$ is defined by $x^{p}-x=z-x^{2} / 2=0$, then $N$ is a finite normal subgroup of $H$ which cannot be defined by two $p$-polynomials in the Frattini coordinates $x$, $z$ of $H$. Thus the assumption of connectivity is also necessary in Corollary 2 .

2. If $G$ and $k$ are as in Theorem 1 and $H$ is any $k$-closed subgroup of codimension one (connected or not), then $H$ can be defined by a single $p$-polynomial in any set of Frattini coordinates. More generally, any $k$-closed subgroup of $G$ containing the Frattini subgroup of $G$ can be defined by $p$-polynomials. Simply note that $\operatorname{codim}_{G} N=\operatorname{codim}_{G / F r(G)} N / F r(G)$ and apply Proposition 2(i) and (ii).

3. An interesting application of Frattini coordinates is the following theorem of Sullivan.

Theorem [6, Theorem 4]. A connected unipotent algebraic group defined over a field of characteristic $p>0$ is conservative if and only if it has dimension one.

Proof. Recall that an algebraic group is conservative if the following condition holds.

Let $W$ be the group of all algebraic group a utomorphisms of $G$. If $f \epsilon$ $K[G]$ then $V_{f}=\left\{w_{*}(f): w \in W\right\}$ is finite dimensional.

By $[2, \S 1]$ this is equivalent to saying that $W$ may be given the structure of an algebraic group in such a way that the natural map $W \times G \rightarrow G$ is a morphism of varieties.

Now let $\operatorname{dim} G>1$ and $K[G]=K\left[x_{1}, \cdots, x_{n}\right]$ where $x_{i}, i=1, \cdots, n$, 
are Frattini coordinates for G. Then it is easily checked (cf. [4, Corollary 2, p. 101]) that the assignments

$$
\begin{array}{ll}
x_{i} \rightarrow x_{i}, & i=1, \cdots, n-1, \\
x_{n} \rightarrow x_{n}+P\left(x_{1}\right), & P \text { a } p \text {-polynomial in } x_{1},
\end{array}
$$

give an automorphism of $G$. In particular $V_{x_{n}}$ is not finite dimensional. It is well known that Aut ${ }_{\mathrm{Alg} \text { group }}\left(G_{a}\right)=G_{m}$ the multiplicative group.

4. If char $K=0$, then with respect to the isomorphism of $G$ with $\underline{A}^{n}$ given by a fixed set of Frattini coordinates, every normal subgroup is a linear subvariety.

5. The converse of Corollary 2 is easily seen to be false. If $G$ is the group of Remark 1 above and $H$ is the subgroup $y=z=0$, it is easily seen that $H$ is not normal in $G$.

\section{REFERENCES}

1. A. Borel, Linear algebraic groups, Benjamin, New York, 1969. MR 40 \#4273.

2. G. Hochschild and G. D. Mostow, Automorphisms of affine algebraic groups, J. Algebra 13 (1969), 535-543.• MR 41 \#315.

3. M. Rosenlicht, Extensions of vector groups by abelian varieties, Amer. J. Math. 80 (1958), 685-714. MR 20 \#5780.

4. - Questions of rationality for solvable algebraic groups over nonperfect fields, Ann. Mat. Pura Appl. (4) 61 (1963), 97-120. MR 28 \#2113.

5. - Nilpotent linear algebraic groups, Sem. Alg. Geom. Topol. (1962/63), vol. 1, Ist. Naz. Alta Mat., Ediz. Cremonese, Rome, 1965, pp. 133-152. MR 32 \#5740.

6. J. B. Sullivan, Automorphisms of affine unipotent groups in positive characteristics, J. Algebra 26 (1973).

7. J. Tits, Lectures on algebraic groups, Lecture Notes, Yale University, New Haven, Conn., 1967.

DEPARTMENT OF MATHEMATICS, UNIVERSITY OF ILLINOIS, URBANA, ILLINOIS 61801 